\title{
Parameter Extraction and Validation of an Electronic and Optical Model for Organic Light-emitting Devices
}

\author{
Beat Ruhstaller ${ }^{*}$, Tilman A. Beierlein ${ }^{* *}$, Roman Gmür ${ }^{*}$, Siegfried Karg ${ }^{* *}$, \\ Heike Riel $^{* *}$, Guido Sartoris *, Hansueli Schwarzenbach ${ }^{*}$ and Walter Riess ${ }^{* * *}$ \\ * Zurich University of Applied Sciences, Center for Computational Physics, \\ Wildbachstrasse 21, 8401 Winterthur, Switzerland \\ ruh@zhwin.ch \\ ** IBM Research GmbH, Säumerstrasse 4, 8803 Rüschlikon, Switzerland \\ wri@zurich.ibm.com
}

\begin{abstract}
Organic light-emitting devices (OLEDs) consist of a stack of multiple thin film layers whose thicknesses influence both the optical and electronic performance. Upon injection and transport, the charge carriers may recombine to form excitons that diffuse and decay radiatively, thus leading to distinct recombination and emission zone profiles. We present systematic combinatorial experiments for parameter extraction and validation of our comprehensive device model. The electronic model is based on drift-diffusion combined with exciton diffusion and decay. The optical part of the model considers the emission to originate from embedded radiative dipoles. We demonstrate the extraction of mobility parameters and energy barriers and validate the optical model using angular emission as well as photoluminescence data.
\end{abstract}

\section{Introduction}

The mechanisms of charge injection, transport and recombination in organic semiconductors are inherently different compared to their inorganic counterparts and demand a specific simulation tool that allows a better understanding and efficient optimization of organic optoelectronics devices and materials. A quantitative understanding of organic light-emitting devices (OLEDs) requires reproducible device fabrication that provides extensive and reliable experimental data for the extraction of material parameters and subsequently for the validation of the models at hand. We therefore employ combinatorial device fabrication, a highly parallel procedure that also allows for efficient device structure optimization [1].

\section{Model}

Numerical simulations have been used in the past to describe the optical [2,3] and the electronic processes in multilayer OLEDs [4,5]. The optical model considers the 
emission to originate from radiative dipoles embedded in a multilayer thin film stack and is solved numerically in one dimension using a transfer matrix method as a function of the viewing angle [6]. The required material parameters consist of the refractive index dispersion and the thickness of the individual layers as well as the emission spectrum of the emitting material, parameters that are easily accessible experimentally. The electronic drift-diffusion model is solved numerically. As for the electronic material parameters, their number is higher and the experimental determination by specific measurements often difficult.

\subsection{Results and Discussion}

We report examples of our attempt to develop a consistent and comprehensive electronic and optical device model by first extracting the material parameters from numerous experiments with simple device structures and few unknowns. In particular, we extract material parameters for the field-dependent mobility from single layer devices assuming space-charge-limited transport (Fig. 1). Secondly, the obtained parameters are then used to simulate more complex multi-layer devices. This approach for model validation is exemplified for the electronic, the excitonic (Fig. 4) and the optical part of the device model (Fig. 2 to 4). For the electronic validation, current-voltage curves and electrostatic potential profiles are used.

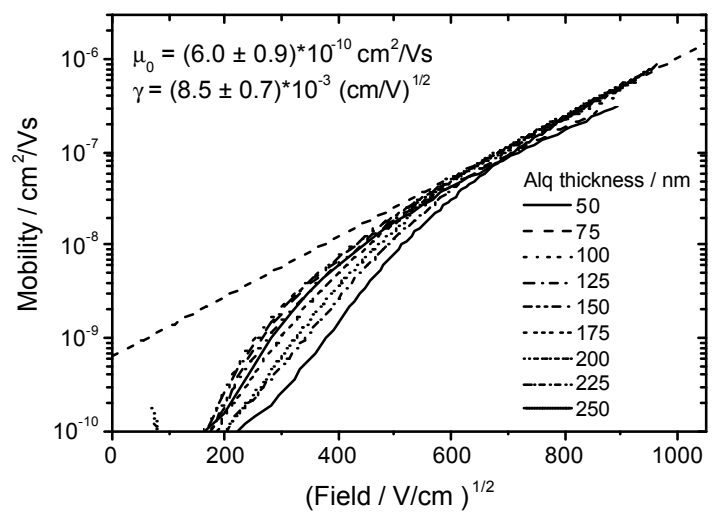

Figure 1: Extraction of the material parameters for field -dependent mobility of electron-only single layer (Alq) devices of various thicknesses from current-voltage curves.

For the optical validation, we compare thickness and polarization dependent (angular) electroluminescence spectra. The angular characteristics of a conventional OLED are illustrated in the example of Fig. 2. Tuning of the angular emission with the thickness of the hole transport layer NPB is shown in a comparison of experimental and simulated data in Fig. 3. In addition, Fig. 4 shows a comparison of a series of thin film photoluminescence experiments that are compared to the results of the optical model [6] and an exciton quenching model. While the optical model gives reliable results for 
thick organic films, the quenching model turns out to be useful for situations where the excitons are in proximity of metal interfaces.
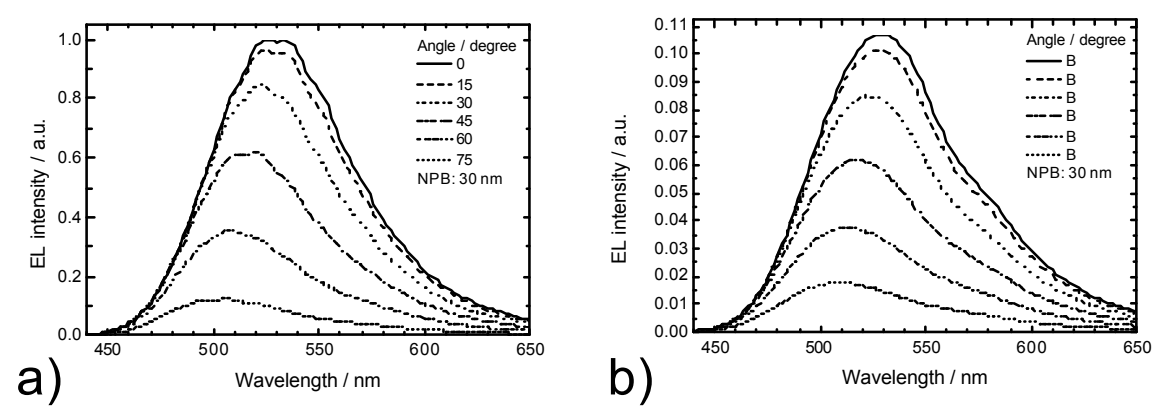

Figure 2: Experimental (a) and simulated (b) emission spectra of a conventional OLED structure $\left(\mathrm{Ni} / \mathrm{CuPc}(15 \mathrm{~nm}) / \mathrm{NPB}(30 \mathrm{~nm}) / \mathrm{Alq}_{3}(50 \mathrm{~nm}) / \mathrm{Ca}(10 \mathrm{~nm}) / \mathrm{Mg}(10 \mathrm{~nm})\right)$ as a function of the viewing angle.

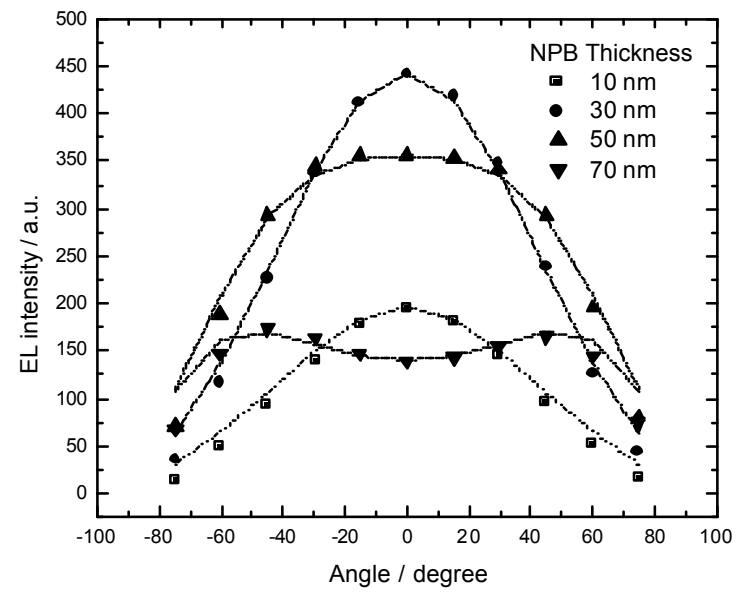

Figure 3: Comparison of experimental (symbols) and simulated (lines) angular luminance for OLEDs of varying NPB layer thickness. The layer structure is the same as for the OLED in Fig. 2.

Our investigations emphasize the importance of thin film effects such as optical interference and electronic confinement. We provide efficient tools for experiment and simulation for a careful design of complex multilayer device structures. 


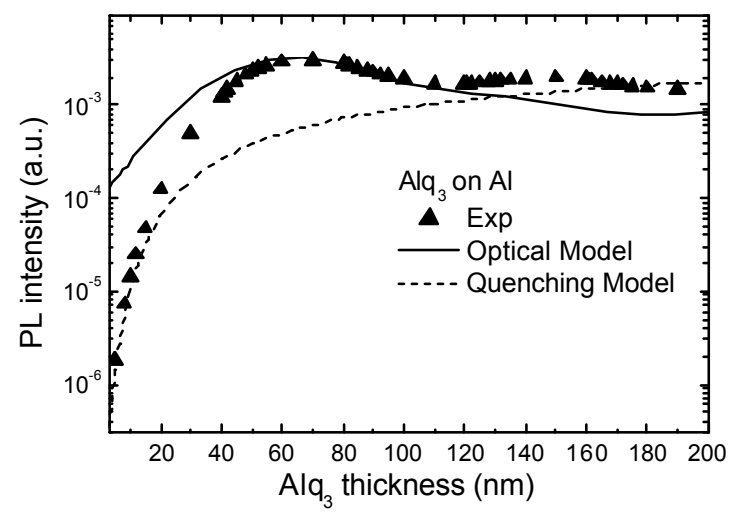

Figure 4: Analysis of photoluminescence experiments with $\mathrm{Alq}_{3}$ thin films on aluminium for investigation of the exciton quenching effect near the metal interface and optical interference at large layer thicknesses .

\section{Summary}

We have presented experiments for parameter extraction and model validation for electronic and optical processes in OLEDs. Charge mobility parameters were extracted from a set of devices with varying thicknesses. For validation of the optical model the angular emission characteristics of a set of OLEDs of varying thickness as well as photoluminescence of thin films was analy zed. The optical part of the model has a reliable and predictive character. Further work is addressed to validate the electronic model using experimental data from multilayer structures.

\section{References}

[1] T. A. Beierlein, B. Ruhstaller, D.J. Gundlach, H. Riel, S. Karg, C. Rost, W. Riess, "Investigation of internal processes in organic light-emitting devices using thin sensing layers", Synth. Met., vol. 138, pp. 213-21, (2003)

[2] H. Riel, S. Karg, T. Beierlein, W. Riess, and K. Neyts, „Tuning the emission characteristics of top-emitting organic light-emitting devices by means of a dielectric capping layer: An experimental and theoretical study“, J. Appl. Phys., vol. 94, pp. 529096, (2003)

[3] J.M. Leger, S.A. Carter, B. Ruhstaller, H.-G. Nothofer, U. Scherf, H Tillman, H.H. Hörhold, "Thickness-dependent changes in the optical properties of PPV- and PF-based polymer light emitting diodes", Phys. Rev. B, vol. 68, 054209 (2003)

[4] B. Ruhstaller, T.A. Beierlein, H. Riel, S. Karg, J.C. Scott, W. Riess, "Simulating electronic and optical processes in multilayer organic light-emitting devices", IEEE Journal on Selected Topics in Quantum Electronics, Optoelectronic Device Simulation, vol. 9 no. 3, pp. 723-732, (2003)

[5] B. Ruhstaller, S.A. Carter, S. Barth, H. Riel, W. Riess, J.C. Scott "Transient and steady state behavior of space charges in multilayer organic light-emitting diodes", J. Appl. Phys., vol. 89, pp. 4575-86, (2001)

[6] ETFOS Emissive Thin Film Optics Simulator, Center for Computational Physics, Switzerland, www.ccp.zhwin.ch, (2004) 